\title{
Orientación y localización como factores de usabilidad: análisis empírico en portales municipales de la Comunidad de Madrid
}

Orientation and localization as usability factors: empirical analysis in the city council web portals of the Community of Madrid

Joaquín B. LóPEZ del RAmo y Pablo R. PRIETo DÁviLA

Universidad Rey Juan Carlos, Camino del Molino S/N, 28943 Fuenlabrada (Madrid), joaquin.lopezdelramo@urjc.es, pablo.prieto@urjc.es

\begin{abstract}
Resumen
Este trabajo analiza la facilidad de orientación y localización y la certidumbre en la navegación como prácticas básicas de usabilidad a través del estudio comparativo de los portales oficiales de los 17 ayuntamientos de Madrid mayores de 50.00 habitantes, realizado durante el año 2008. El objetivo básico es medir los niveles de eficacia, detectar tendencias, comparar y catalogar los componentes de estas rutinas de usabilidad. Los resultados indican que el factor facilidad de localización u orientación se acerca más al estándar ideal que el factor certidumbre en la navegación. Los indicadores con mejor tratamiento son: rotulación, motores de búsqueda, etiquetado y legibilidad. Se obtienen las siguientes conclusiones: a) El tratamiento general de estos factores de usabilidad posee unos niveles aceptables, si bien es desigual en sus componentes individuales. b) Para el usuario resulta más fácil saber dónde está que a dónde puede ir. c) El usuario no encontraría grandes diferencias entre estos los portales analizados.
\end{abstract}

Palabras clave: Usabilidad. Localización y orientación. Navegación con certidumbre. Webs municipales. Comunidad de Madrid.

\section{Introducción}

Este trabajo se enmarca en el ámbito del proyecto de investigación "Contenidos, arquitectura y participación ciudadana en las web de los ayuntamientos de la Comunidad de Madrid mayores de 50.000 habitantes", del Programa de Creación y Consolidación de Grupos de Investigación 2007- URJC-CAM.

Este proyecto de investigación tiene como premisa básica realizar una evaluación exhaustiva respecto de las características estructurales y operativas de los sitios web de ayuntamientos de la Comunidad de Madrid. Se trata de determinar empíricamente la idoneidad de estos factores, considerando los portales web como pro-

\begin{abstract}
Usability analysis of the official web portals of the 17 city councils of the Community of Madrid with more than 50.000 inhabitants during the year 2008. The basic objectives are measuring the efficiency levels, detecting trends, comparing and cataloguing the components of their usability routines. The results show that the ease of localization and orientation is closer to the ideal standard than certainty in navigation. The indicators with better results were: lettering, search engines, labelling and legibility. The main conclusions are: a) The general treatment of these usability factors is at acceptable levels, but there are differences in the individual components. b) It is easier for the user to know where he is than where he can go. c) The users would not find great differences between the analyzed web portals.
\end{abstract}

Keywords: Usability. Localization and orientation. Web navigation with certainty. City council websites. Community of Madrid.

ducto cuyo sentido último es poner a disposición del usuario un conjunto de información y servicios a los que éste pueda acceder de forma clara, coherente, intuitiva y ágil.

Desde que en 1999 Jakob Nielsen escribiera el libro Designing Web Usability: The Practice of Simplicity y abriera el camino del diseño web "centrado en el usuario" se ha avanzado y escrito mucho en torno a la usabilidad. El diseño web centrado en el usuario ha conducido a la expresión de enunciados, casi epigramas, como los expresados por Garret: “Your users aren't stupid. Why does your site make them feel that way?" ("Tus usuarios no son estúpidos. ¿Por qué tu sitio web les hace sentir así?") (2002) o el 
propio título del libro de Steve Krug: "Don't Make Me Think!" (“¡No me hagas pensar!”) (2000).

En esta línea, la de la usabilidad como diseño centrado en el usuario, este trabajo aborda el estudio de dos factores fundamentales de la usabilidad:

- El primer factor es la orientación y localización, que el usuario sepa dónde está. Patrick J. Lynch y Sara Horton (2004, p. 21) lo expresan en los siguientes términos:

El principal problema de interfaz en los sitios es la pérdida del sentido de la orientación dentro de la organización local de la información.

- El segundo factor es la certidumbre en la navegación, que el usuario sepa dónde puede ir. Steve Krug (2006, p. 41) aborda la cuestión desmintiendo uno de los mitos de la usabilidad, la distancia de clic, en favor de la certidumbre de clic:

Lo que realmente importa no es el número de clics que tengo que hacer para alcanzar lo que quiero (si bien existen límites), sino la dificultad en la elección de ese clic (el esfuerzo de pensamiento y la incertidumbre sobre si la elección tomada es la correcta).

Sobre la base de las proposiciones anteriores, el presente estudio se orienta en torno a cuatro objetivos: a) catalogar los factores básicos que inciden en la orientación, localización y certidumbre de navegación; b) constatar de forma empírica la incidencia individual de los mismos, sus fortalezas y carencias, por medio de un modelo de análisis original creado específicamente para esta investigación; c) comprobar si el tratamiento de esta práctica de usabilidad en portales institucionales alcanza unos niveles aceptables; y d) detectar posibles tendencias o desigualdades en dicha práctica, tanto a escala general (de todos los portales analizados) como a escala individual (de un portal concreto).

\section{Metodología}

El análisis se lleva a cabo a partir de una muestra que incluye una página representativa de cada uno de los tres niveles jerárquicos básicos de los 17 portales implicados en el estudio, dado el parecido estructural-visual que se presupone a las páginas pertenecientes a un mismo nivel. En todo caso, la existencia de dicha coherencia entre las páginas de cada nivel ha sido previamente testada, eligiéndose una página representativa de cada nivel por portal. De haber incoherencia, se determinaron los diferentes estilos estructurales-visuales de páginas presentes en un mismo nivel del portal y se escogió una página igualmente representativa de cada estilo presente en dicho nivel del Sitio. El análisis fue realizado entre los meses de enero y julio del año 2008.

Coincidiendo con los planteamientos de Lynch y Horton (2004, p. 91 y ss.), el usuario necesita ayudas claras a la navegación. Tanto los citados autores como Steve Krug (2006, p. 51 y ss.) sistematizan los indicadores que ayudan al usuario a saber dónde está y a dónde puede ir.

Desde el punto de vista metodológico, se lleva a cabo un análisis de contenido en el son consideradas dos categorías estrechamente relacionadas entre sí:

a) Facilidad de localización y orientación ofrecida por el portal, tomando lo expuesto por Lynch y Horton (2004) sobre el particular. Su objetivo es evaluar si se indica correcta y claramente al usuario a dónde puede ir y si se le muestra la estructura y la organización del Sitio.

b) Grado de certidumbre en la navegación, en el sentido indicado por Krug (2006), es decir, indagar si el usuario va a tener le certeza de lo que va a encontrar detrás de cada clic, que implica conocer de forma clara por parte de éste a dónde puede ir a partir del punto de la web donde se encuentra en ese momento.

\begin{tabular}{ll}
\hline 1. Orientación y localización & \\
\hline Indicador & Valores \\
\hline $\begin{array}{l}\text { 1.1. Existencia de Mapa web } \\
\text { 1.2. Empleo de índices de contenido o } \\
\text { submenús }\end{array}$ & Si/No \\
\hline $\begin{array}{l}\text { 1.3. Índices o directorios como } \\
\text { motores de búsqueda }\end{array}$ & Sí/No \\
\hline $\begin{array}{l}\text { 1.4. Existencia de buscador interno } \\
\text { 1.5. Empleo de migas de pan }\end{array}$ & Sí/No \\
\hline $\begin{array}{l}\text { 1.6. Separa áreas de navegación con } \\
\text { distinta funcionalidad o destino }\end{array}$ & Sí/No \\
\hline 2. Certidumbre en la navegación & \\
\hline $\begin{array}{l}\text { Indicador } \\
\text { 2.1. Etiquetas ALT descriptivas }\end{array}$ & Valores \\
$\begin{array}{l}\text { 2.2. Intuitividad de iconos con link } \\
\text { 2.3. Identificación expresa de vínculos } \\
\text { externos }\end{array}$ & Sí/No \\
$\begin{array}{l}\text { 2.4. Rótulos con lenguaje cercano al } \\
\text { usuario }\end{array}$ & Sí/No \\
2.5. Redundancia entre etiquetas & Sí/No \\
\hline 2.6. Correcta legibilidad del etiquetado & Sí/No \\
\hline
\end{tabular}

Tabla I. Indicadores de la ficha de análisis

Las preguntas esenciales de la investigación se corresponden en este caso con los dos aspectos o categorías generales de usabilidad evaluadas, que desde el punto de vista del usuario 
implican básicamente dos cosas: saber dónde está y saber dónde puede ir. Las dos categorías se desglosan en variables individuales que actúan como indicadores de cumplimiento correcto de ambos factores de usabilidad.

La ficha de análisis utilizada ha sido elaborada por el equipo de investigación del proyecto y se describe en la Tabla I, en la página anterior.

Esta ficha de análisis se aplicó de forma exhaustiva a todas las páginas web de la muestra, seleccionadas con los criterios antes expuestos. Una vez realizado el vaciado de las mismas, los datos obtenidos se guardaron en la matriz informática realizada al efecto. Dichos datos respondían a los valores de los indicadores de la matriz de análisis correspondientes al nivel estructural específico de cada una de las páginas examinadas en los diferentes portales, desde nivel 1 (página principal o home) al nivel 3.

A partir de los datos primarios obtenidos, y siguiendo a Igartua (2007), se lleva a cabo una exploración de tipo univariado que permite la obtención de frecuencias, porcentajes y tendencias de los diferentes indicadores, en correspondencia con los objetivos del trabajo. Asimismo, se realiza una lectura cruzada para comparar entre sí el valor de los diferentes indicadores en cada uno de los portales objeto de estudio. Se ofrecen separadamente resultados totales, de los dos factores o categorías de usabilidad analizadas, y específicos, es decir, de las seis variables individuales contempladas en cada uno.

Para evaluar los resultados se creó por parte del equipo investigador (panel de expertos) un modelo de referencia ideal que incluyó las mismas categorías e indicadores y que se utiliza como patrón de referencia para contrastar grado de cumplimiento de los estándares idóneos de usabilidad en las páginas representativas de los tres niveles estructurales de cada portal.

\section{Resultados}

3.1. Análisis univariado: frecuencia y porcentaje de categorías e indicadores de usabilidad

\section{Categoría 1: Orientación y localización}

Los resultados obtenidos reflejan que el valor medio global de todos los portales posee un índice de aproximación del $75^{\prime} 8 \%$ respecto al tratamiento ideal (Tabla II) de este factor de usabilidad. El valor medio de todos los portales es 12 ' 9 y el valor del modelo ideal 17 .

En un nivel de detalle más preciso, el desglose de los resultados de esta categoría por indica- dores (Tabla III) muestra las siguientes tendencias, que se presentan aquí en orden descendente según su porcentaje de acercamiento a los valores ideales:

- Existencia de buscador interno: se da en el $90 \%$ de las páginas analizadas.

- Separación de barras y elementos de navegación con distinta funcionalidad o destino: registra una frecuencia de empleo del $90 \%$ de la muestra.

- Existencia de mapa web funcional (con vínculos): le corresponde el 82'3 \% de utilización.

- Empleo de índices de contenido y submenús: se da en el $80 \%$ de la muestra.

- Empleo de migas de pan: se registra en el $70 \%$ de la muestra.

- Empleo de índices alfabéticos o directorios como motores de búsqueda: es el valor que posee un menor porcentaje de aproximación al modelo ideal en esta categoría: el $40 \%$.

\section{Categoría 2: Certidumbre en la navegación}

En el caso de la segunda categoría de usabilidad considerada en este trabajo, la certidumbre en la navegación (Tabla II), el resultado medio se cifra en el 52 ' $2 \%$ respecto al modelo ideal. El valor medio de todos los portales es 14 '1 y el valor del modelo ideal 27 . Ello supone una diferencia negativa de más de 20 puntos porcentuales respecto al tratamiento del otro factor analizado: la orientación y localización.

Los resultados obtenidos en las variables componentes de esta categoría aparecen listados en la Tabla III. Mencionadas en orden decreciente de acercamiento al modelo ideal, se distribuyen de la siguiente manera:

- Rotulación con lenguaje cercano al usuario: se produce en el $100 \%$ de los casos, siendo la variable con mejores resultados de todo el estudio.

- Legibilidad correcta del etiquetado: posee respuesta afirmativa en el 92 '1\% de los casos analizados.

- Etiquetas ALT descriptivas del destino en vínculos: se constata su uso en el 71 '3\% de la muestra.

- Buena intuitividad de los iconos gráficos que poseen link: se registra en el $62,5 \%$ de la muestra, teniendo en cuenta que sólo se evalúa en aquellas páginas que poseen iconos con link, lo que ocurre únicamente en 8 de los 17 portales estudiados. 
- Redundancia o parecido entre etiquetas: es una mala praxis de usabilidad que se da en el 66 '6\% de la muestra, lo que a la inversa implica que la buena praxis sólo se produce en el $33^{\prime} 4 \%$ de los casos.

- Identificación explícita de los links externos: es el indicador con peor resultado del estudio, pues su acercamiento al modelo ideal se cifra en el 2'1\%. Asimismo, este indicador sólo se evalúa en aquellas páginas que poseen links externos, lo que ocurre sucede en 16 de los 17 portales analizados.

\subsubsection{Cómputo global de las dos categorías de análisis}

Como corolario de los resultados mencionados en este epígrafe, se constata que el factor orientación y localización presenta un grado de acercamiento a los niveles de usabilidad ideales notablemente más elevado que el factor certidumbre en la navegación. Asimismo, los resultados de los indicadores específicos de ambos factores presentan más homogeneidad en el primer factor que en el segundo, donde predomina una mayor desigualdad.

Consideradas ambas categorías de usabilidad en su conjunto, el factor de aproximación general a los niveles ideales es del $64 \%$.

\subsection{Referencias cruzadas: categorías e} indicadores de usabilidad por portales

\subsubsection{Categoría 1: Tratamiento de la Orienta- ción y localización por portales}

Como se indicó en el epígrafe anterior, en esta categoría del análisis la media global arroja un valor de 12 '9 sobre un ideal de 17 (75'8\% de aproximación). Por encima de este porcentaje aparecen los Sitios institucionales de nueve ayuntamientos (Tabla II): Alcobendas, Collado Villalba, Fuenlabrada, Getafe, Majadahonda, Parla, Pozuelo, San Sebastián de los Reyes y Valdemoro. En dos de ellos, Majadahonda y Pozuelo, el grado de cumplimiento respecto al modelo ideal es del $100 \%$. Muy cercanos a este índice, con un $94 \%$ de aproximación, están las webs de Alcobendas y Fuenlabrada.

Los portales que figuran por debajo de la media general en esta categoría del análisis son ocho: Alcalá de Henares, Alcorcón, Coslada, Leganés, Móstoles, Rivas, Las Rozas y Torrejón. Aún estando a nivel inferior de la media, los dos portales que están más próximos a la media global son los de Alcalá y Leganés, que cumplen en un 70 ' $5 \%$ el modelo ideal, lo cual es un indicativo bastante favorable. El índice más bajo de cumplimiento frente al valor ideal, se registra en la web de Móstoles, con un 52'9\%.

Como resultado de las anteriores cifras, la diferencia entre los valores obtenidos en esta categoría de usabilidad por los portales con mejores índices (100\%) y peores (52'9\%) es del $41{ }^{\prime} 1 \%$. No obstante, salvo esta desviación entre extremos, la tónica general presenta desigualdades mucho menos acentuadas.

\subsubsection{Categoría 2: Tratamiento de la Certidum- bre en la navegación por portales}

Esta categoría presenta un menor grado de acercamiento al modelo ideal, 14 ' 1 sobre 27 $(52 ' 2 \%)$. Como en el caso anterior, dicho resultado es el global resultante de la media de los parciales correspondientes a cada portal analizado.

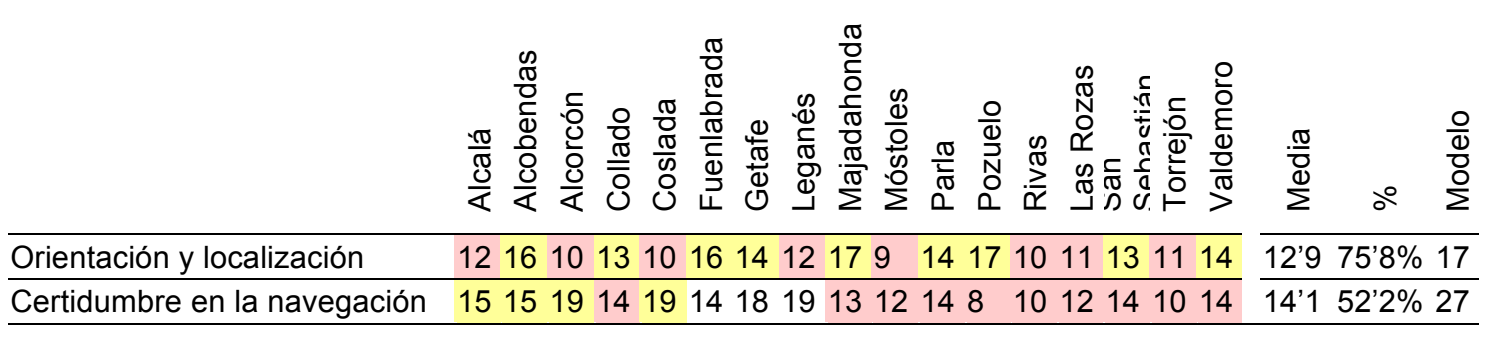

Valores por encima de la media

Valores por debajo de la media

Tabla II. Frecuencia y porcentaje de categorías de análisis por portales

El desglose particular de los resultados (Tabla II) permite observar que sólo seis de los 17 portales analizados sobrepasan el valor medio de la categoría: Alcalá, Alcobendas, Alcorcón, Cos- lada, Getafe y Leganés. De ellos, los que registran un valor más elevado son Alcorcón, Coslada y Leganés, todos con 19, lo que porcentualmente representa un índice de acercamiento al 
modelo ideal del 70 ' $3 \%$, es decir, casi 20 puntos de diferencia favorable respecto a la media del conjunto de portales. Les sigue por debajo la web de Getafe, que posee un índice de aproximación al ideal en esta categoría de usabilidad del 66 ' $6 \%$. Por debajo de la media global figuran 11 portales: Collado Villalba, Fuenlabrada, Majadahonda, Móstoles, Parla, Pozuelo, Rivas, las Rozas, San Sebastián de los Reyes, Torrejón y Valdemoro. De todos ellos, cinco se quedan con un valor muy próximo a la media global, $14 \mathrm{de}$ éstos frente a 14'1 de aquella, lo que traducido a porcentajes representa un acercamiento del $51^{\prime} 8 \%$ al valor ideal. Se trata de los portales municipales de Collado Villalba, Fuenlabrada, Parla, San Sebastián de los Reyes y Valdemoro. El valor más bajo de los obtenidos en esta categoría de usabilidad corresponde al portal de Pozuelo, con un acercamiento del 29 '6\% al modelo ideal.

Así pues, la diferencia porcentual entre los portales que presentan mejores $(70,3 \%)$ y peores índices de cumplimiento $(29 \% 6)$ en esta categoría es de un 40 ' $7 \%$. No obstante, como ocurre en la categoría anterior, la mayor parte de las webs analizadas arroja unos resultados bastante uniformes, sin diferencias tan acentuadas entre ellos.

\subsubsection{Cómputo global por portales}

La contemplación global de los datos del estudio tomando como referencia los diferentes portales (Tabla II) permite constatar otros dos aspectos relevantes: a) Únicamente hay dos portales que están por encima de la media en las dos categorías del análisis: los de los ayuntamientos de Alcorcón y de Getafe. b) Hay cuatro portales cuyos resultados están por debajo de la media en las dos categorías: Móstoles, Rivas, Las Rozas y Torrejón.

\subsubsection{Cómputo de indicadores individuales por portales}

Para la correcta interpretación de los resultados expuestos en este epígrafe, debe tenerse en cuenta el significado de los valores de codificación de los diferentes indicadores. En tal sentido se señala que en todos ellos, salvo en tres que con posterioridad se identifican, el valor ideal es 3 , lo que significa una respuesta positiva en las páginas de los tres niveles jerárquicos de cada portal analizado, pues son indicadores dicotómicos (repuesta Sí/No). Las excepciones a esta pauta son los siguientes indicadores, que se presentan en la Tabla III.

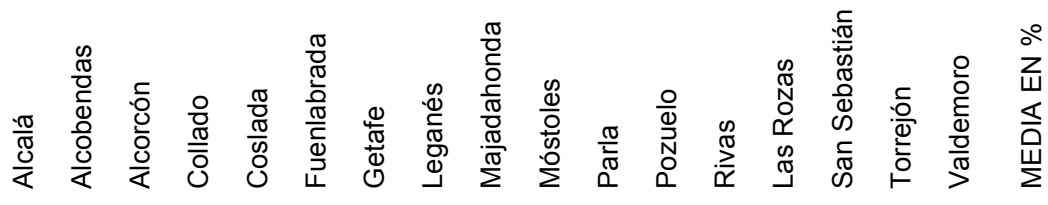

Categoría 1: Orientación y localización

\begin{tabular}{|c|c|c|c|c|c|c|c|c|c|c|c|c|c|c|c|c|c|c|}
\hline Existencia de mapa web (1) & 3 & 3 & 3 & 0 & 3 & 3 & 3 & 3 & 3 & 3 & 3 & 3 & 0 & 0 & 3 & 3 & 3 & $82^{\prime} 3$ \\
\hline Índices de contenido y/o submenús (1) & 2 & 3 & 2 & 3 & 1 & 3 & 1 & 2 & 3 & 1 & 3 & 3 & 3 & 3 & 3 & 2 & 3 & 80 \\
\hline Directorios como motor de búsqueda (1) & 1 & 2 & 0 & 3 & 1 & 2 & 2 & 1 & 3 & 0 & 0 & 3 & 2 & 0 & 1 & 0 & 0 & \\
\hline Existe Buscador interno (1) & 3 & 3 & 1 & 3 & 0 & 3 & 3 & 3 & 3 & 3 & 3 & 3 & 3 & 3 & 3 & 3 & 3 & \\
\hline Empleo de Migas de pan (2) & 0 & 2 & 2 & 2 & 2 & 2 & 2 & 0 & 2 & 2 & 2 & 2 & 0 & 2 & 0 & 0 & 2 & 10 \\
\hline Separa barras por funcionalidad (1) & 3 & 3 & 3 & 2 & 3 & 3 & 3 & 3 & 3 & 0 & 3 & 3 & 2 & 3 & 3 & 3 & 3 & \\
\hline \multicolumn{19}{|l|}{ Categoria 2: Certidumbre en la navegación } \\
\hline Etiquetas ALT descriptivas (3) & 15 & 10 & 10 & 9 & 10 & 15 & 10 & 15 & 14 & 9 & 15 & 4 & 3 & 9 & 15 & 5 & 15 & 71 \\
\hline Intuitivi & 0 & 3 & 3 & 2 & 3 & 0 & 1 & 3 & 0 & 0 & 0 & 0 & 0 & 0 & 0 & 0 & 0 & 62 \\
\hline Identific & 0 & 0 & 0 & 0 & 0 & 0 & 0 & 0 & 0 & 0 & 0 & 0 & 1 & 0 & 0 & 0 & 0 & \\
\hline Rótulos con lenguaje cercan & 3 & 3 & 3 & 3 & 3 & 3 & 3 & 3 & 3 & 3 & 3 & 3 & 3 & 3 & 3 & 3 & 3 & \\
\hline Redundancia entre etiquetas (5) & 2 & 1 & 1 & 0 & 0 & 3 & 0 & 1 & 3 & 3 & 3 & 3 & 3 & 2 & 3 & 3 & 3 & 66 \\
\hline Legibilidad correcta del etiquetad & 3 & 3 & 3 & 3 & 3 & 3 & 3 & 3 & 3 & 3 & 3 & 2 & 0 & 3 & 3 & 3 & 3 & 52 \\
\hline $\mathrm{N}^{\circ}$ Indicadores con valores ideales & 6 & 7 & 5 & 6 & 6 & 7 & 6 & 7 & 7 & 4 & 7 & 6 & 4 & 5 & 7 & 5 & 7 & \\
\hline
\end{tabular}

(1) El valor ideal de los indicadores es 3 , lo que significa una respuesta positiva en las páginas de los tres niveles jerárquicos de cada portal analizado.

(2) Este indicador no se analiza en las páginas de primer nivel (Home) por carecer de sentido, por tanto, su valor ideal es 2.

(3) El valor ideal para este indicador es 15 , pues tiene 5 respuestas posibles por cada uno de los tres niveles de la web, siendo la más positiva la 5 (en todos los casos)

(4) Se evalúa únicamente en las páginas que utilizan este recurso: iconos con vínculos.

(5) El valor ideal es 0 , ya que la mejor práctica de usabilidad es que no exista ninguna redundancia entre etiquetas.

Tabla III. Frecuencias y porcentajes de los indicadores por portales 
- Empleo de migas de pan: no se analiza en las páginas de primer nivel (Home) por carecer de sentido; por tanto, su valor ideal es 2.

- Etiquetas ALT descriptivas del destino: El valor ideal para este indicador es 15 , pues tiene 5 respuestas posibles por cada uno de los tres niveles de la web, siendo la más positiva la 5 (empleo en todos los casos).

- Redundancia entre etiquetas: El valor ideal es 0 , ya que la mejor práctica de usabilidad es que no se produzca esta circunstancia.

- Intuitividad de iconos con link: Se evalúa únicamente en las páginas que utilizan este recurso.

Sentadas estas pautas, y en base a la lectura cruzada de los indicadores (filas) con los portales de los diferentes ayuntamientos (columnas) de la Tabla III, en la página anterior, se aprecia que es posible jerarquizar las webs municipales en relación al número de indicadores con valores ideales que poseen. Ello otorga una visión global de los resultados del estudio, al integrar los indicadores de las dos categorías de usabilidad consideradas.

Así, en primer lugar aparecen los portales de los ayuntamientos de Alcobendas, Fuenlabrada, Leganés, Majadahonda, Parla, San Sebastián de los Reyes y Valdemoro, todos ellos con 7 indicadores que registran el valor máximo, lo que teniendo en cuenta que el total de indicadores considerados en el estudio es 12 , equivale al 58 ' $3 \%$ de acercamiento al modelo ideal. Con 6 indicadores en valor óptimo, equivalente al $50 \%$ de aproximación al modelo, están los sitios web de Alcalá, Collado Villalba, Coslada, Getafe y Pozuelo. En un nivel inferior están los portales de Alcorcón, Las Rozas y Torrejón, que registran 5 indicadores en valores máximos, el $41 ' 6 \%$. Los porcentajes más bajos de la muestra corresponden a los sitios oficiales de Móstoles y Rivas, ambos con un 33'3\% de aproximación al ideal al tener sólo cuatro indicadores con valor óptimo.

Para todos los portales en su conjunto, la media global de acercamiento a los valores ideales es de 6 indicadores, equivalente al $50 \%$.

\section{Conclusiones}

Las conclusiones fundamentales del trabajo son:

(a) La orientación, localización y certidumbre son factores de usabilidad que poseen unos niveles aceptables en los portales estudiados, si bien sus indicadores individuales reciben un tratamiento desigual.

(b) Los factores relativos a la certidumbre en la navegación están menos conseguidos que el resto de los directamente relacionados con la localización dentro de la web, por lo que para el usuario resulta más fácil saber dónde está que a dónde puede ir. El modelo de análisis creado para la investigación arroja resultados coherentes, con información verificable y reproducible, por lo que se considera validado.

(c) La mayoría de los portales presenta mejores niveles en los mismos aspectos: buena rotulación y legibilidad, existencia de mapa web, buscador interno y etiquetas descriptivas.

(d) Los portales con mejores niveles de acercamiento al patrón ideal de los factores de usabilidad estudiados son los de Alcobendas y Getafe.

\section{Recomendaciones}

El trabajo abre otras perspectivas para la posible ampliación del mismo objeto de estudio, tanto más habida cuenta de la escasez de investigaciones empíricas existente.

Podría profundizarse más incorporando al modelo de análisis otros factores que inciden en la facilidad de orientación del usuario, tales como los relacionados con el diseño gráfico (tipografía, colores, formas) o cruzarse con otros indicadores de navegación, arquitectura o diseño de superficie de página. El modelo también es susceptible de ser aplicado a portales de cualquier otro objeto o ámbito temático.

\section{Referencias}

Garret, Jesse James (2002). The Elements of User Experience: User-Centered Design for the Web. Berkeley: New Riders, 2002.

Igartua, Juan.José. (2007). Métodos cuantitativos de investigación en comunicación. Barcelona: Bosch, 2007.

Krug, Steve (2000). Don't Make Me Think: A Common Sense Approach to Web Usability, Berkeley, New Riders, 2000. Las citas están tomadas de la edición española: Krug, Steve (2006). No me hagas pensar: Una aproximación a la usabilidad en la web, Madrid, Pearson Educación, 2006.

Lynch, Patrick J. y Horton, Sara (2004). Manual de estilo web: Principios de diseño básico para la creación de sitios web. Barcelona, 2004.

Nielsen, Jakob (1999). Designing Web Usability: The Practice of Simplicity. Berkeley: New Riders, 1999. 